\title{
An adaptive mealtime bolus calculator to minimize the effects of inaccurate carbohydrate counting
}

Cite as: AIP Conference Proceedings 2116, 250011 (2019); https://doi.org/10.1063/1.5114251

Published Online: 24 July 2019

Carlos Abreu (D), Francisco Miranda (D), and Paula Felgueiras (D)

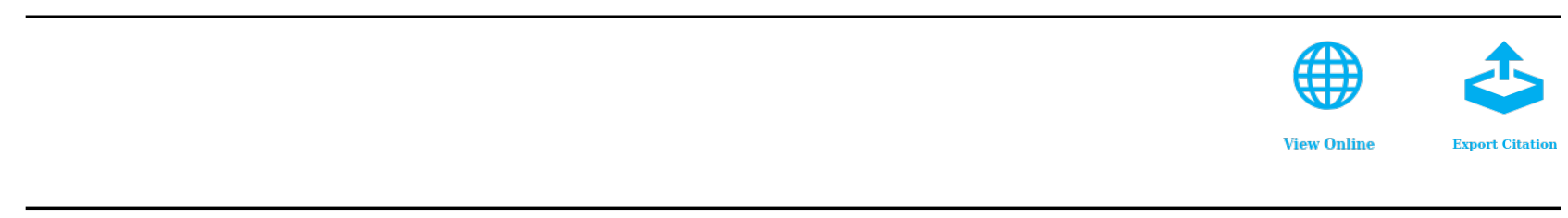

\section{Conference Proceedings}

Get $30 \%$ off all print proceedings!

\section{Enter Promotion Code PDF30 at checkout}




\title{
An Adaptive Mealtime Bolus Calculator to Minimize the Effects of Inaccurate Carbohydrate Counting
}

\author{
Carlos Abreu ${ }^{1,2, a)}$, Francisco Miranda ${ }^{1,3}$ and Paula Felgueiras ${ }^{1,4}$ \\ ${ }^{1}$ Instituto Politécnico de Viana do Castelo, Viana do Castelo, Portugal \\ ${ }^{2}$ CMEMS-UMinho, Universidade do Minho, Braga, Portugal \\ ${ }^{3}$ CIDMA, Universidade de Aveiro, Aveiro, Portugal \\ ${ }^{4}$ Unidade Local de Saúde do Alto Minho, Hospital de Santa Luzia, Viana do Castelo, Portugal \\ ${ }^{a)}$ Corresponding author: cabreu @estg.ipvc.pt
}

\begin{abstract}
Evidence suggests that accurate carbohydrate counting along with self-monitoring of blood glucose is the key to a successful diabetes management, in particular for patients on intensive insulin regimens. However, despite its benefits, accurate carbohydrate counting is a complex, difficult, time-consuming, and error-prone task for most patients. Several studies show that most patients frequently estimate the carbohydrate content of meals within an error of about $10-15 \mathrm{~g}$ of the real value. In addition, fearing hypoglycemic events, patients frequently underestimate the carbohydrate content of meals and, consequently, they have high levels of HbA1C. Therefore, is important to avoid the consequences of incorrect carbohydrate counting in order to improve the patient's glycemic control. To that end, this work presents an adaptive mealtime bolus calculator that uses the patient's glycemic data to dynamically adjust the mealtime bolus and counterbalance the negative effects of inaccurate carbohydrate counting.
\end{abstract}

\section{INTRODUCTION}

Patients on intensive insulin regimens use carbohydrate counting and self-monitoring of blood glucose to find the insulin dose required for each meal [1,2]. The correct calculation of the proper insulin bolus has two parts: the prandial bolus, and the correction bolus. Regarding the prandial bolus, it mainly depends on the ability of each patient to accurately estimate the carbohydrate content of each meal. Indeed, the accuracy of carbohydrate counting is crucial to avoid postprandial hyperglycemic and hypoglycemic events [3]. As reported by Smart et al in [4, 5], an error between 10-20 g, when estimating the carbohydrate content of a meal containing $60 \mathrm{~g}$ of carbohydrates may result in dysglycemia. To worsen the situation, such errors are very frequent. Smart et al investigated how accurately children and their caregivers estimate the carbohydrate content of meals and found that $73 \%$ of meals were within $10-15 \mathrm{~g}$ of real carbohydrate content [6]. On its turn, Bishop et al reported that only $23 \%$ of the adolescents with type 1 diabetes estimate the carbohydrate content of meals within $10 \mathrm{~g}$ of the true amount [7]. In this context, this work proposes an adaptive mealtime bolus calculator able to minimize the adverse effects of inaccurate carbohydrate counting. The proposed bolus calculator makes use of the estimated carbohydrate content of each meal and the average postprandial glycemia to dynamically adjust the insulin bolus in order to improve the glycemic control of the patient.

\section{MATHEMATICAL METHODS}

Patients on intensive insulin regimens using carbohydrate counting and self-monitoring of blood glucose calculate their insulin dosing according to the following equation:

$$
B=\left(\frac{C H O}{I C R}+\frac{G-G_{T}}{I S F}\right) K-I O B,
$$

where $B$ is the required bolus in $\mathrm{U}$ (Units of Insulin), $C H O$ is the amount of carbohydrates to be consumed in $\mathrm{g}, I C R$ is the insulin-to-carb ratio in $\mathrm{g} / \mathrm{U}$ (n.b., $I C R>0$ ), $G$ is the premeal blood glucose in $\mathrm{mg} / \mathrm{dL}, G_{T}$ is the blood glucose

International Conference of Numerical Analysis and Applied Mathematics (ICNAAM 2018)

AIP Conf. Proc. 2116, 250011-1-250011-4; https://doi.org/10.1063/1.5114251

Published by AIP Publishing. 978-0-7354-1854-7/\$30.00 
target in $\mathrm{mg} / \mathrm{dL}, I S F$ is the insulin sensitivity factor in $\mathrm{mg} / \mathrm{dL} / \mathrm{U}$ (n.b., $I S F>0$ ), $K$ is a correction factor used to compensate for the effects of some infection, physical activity or carbohydrates intake, and IOB (Insulin-on-Board) is an estimate of the insulin still active in the body from previous bolus in U [1]. In the following analysis, we will consider $I O B=0$, i.e., the time between meals is considered to be larger than the duration of the insulin action, and there is no insulin stacking. Furthermore, we also consider that patient's ICR and $I S F$ are physiologically appropriate.

Let us consider the carbohydrate content of each meal as $C H O=C \hat{H} O \pm \triangle C H O$, where $C \hat{H} O$ is an estimate of $\mathrm{CHO}$ with an absolute error $\triangle \mathrm{CHO}>0$. The remaining variables of Equation 1 are considered to be error-free. In such circumstances, and without any correction (i.e., $K=1$ ) the absolute error of the bolus, $\Delta B$, as a consequence of $\triangle C H O$ is given by $\Delta B=|B-\hat{B}|$, where: $B=C H O / I C R+\left(G-G_{T}\right) / I S F$ and $\hat{B}=C \hat{H} O / I C R+\left(G-G_{T}\right) / I S F$. As a result, $\Delta B$ will act as an unplanned correction bolus with a negative impact on the patients' glycemic control causing an undesired variation on the postprandial glycemia, $G_{\text {postprandial }}$, given by:

$$
\Delta G_{\text {postprandial }}=\left|G_{\text {postprandial }}-G_{T}\right|=\Delta B \cdot I S F=\frac{I S F}{I C R} \Delta C H O
$$

Therefore, to correct the bolus it is necessary to find the value of $K$ so that:

$$
\begin{aligned}
& \hat{B} \cdot K=B \\
& \Leftrightarrow \quad\left(\frac{C \hat{H} O}{I C R}+\frac{G-G_{T}}{I S F}\right) K=\frac{C H O}{I C R}+\frac{G-G_{T}}{I S F} \\
& \Leftrightarrow \quad \frac{C \hat{H} O \cdot I S F+\left(G-G_{T}\right) I C R}{I C R \cdot I S F} K=\frac{C H O \cdot I S F+\left(G-G_{T}\right) I C R}{I C R \cdot I S F} \\
& \Leftrightarrow \quad K=\frac{C H O \cdot I S F+\left(G-G_{T}\right) I C R}{C \hat{H} O \cdot I S F+\left(G-G_{T}\right) I C R} \\
& \Leftrightarrow \quad K=\frac{C \hat{H} O \pm \Delta C H O+\left(G-G_{T}\right) \frac{I C R}{I S F}}{C \hat{H} O+\left(G-G_{T}\right) \frac{I C R}{I S F}}, \quad \text { using: } C H O=C \hat{H} O \pm \Delta C H O \\
& \Leftrightarrow \quad K=1 \pm \frac{\Delta C H O}{C \hat{H} O+\left(G-G_{T}\right) \frac{I C R}{I S F}} \\
& \Leftrightarrow \quad K=1 \pm \frac{\left|G_{\text {postprandial }}-G_{T}\right| \frac{I C R}{I S F}}{C \hat{H} O+\left(G-G_{T}\right) \frac{I C R}{I S F}}, \quad \text { from Eq. 2: } \Delta C H O=\left|G_{\text {postprandial }}-G_{T}\right| \frac{I C R}{I S F} .
\end{aligned}
$$

Finally,

$$
K=\left\{\begin{array}{lll}
1+\frac{\left|G_{\text {postprandial }}-G_{T}\right| \frac{I C R}{I S F}}{C \hat{H} O+\left(G-G_{T}\right) \frac{I C R}{I S F}} & \text { if } & G_{\text {postprandial }}>G_{T} \\
1-\frac{\left|G_{\text {postprandial }}-G_{T}\right| \frac{I C R}{I S F}}{C \hat{H} O+\left(G-G_{T}\right) \frac{I C R}{I S F}} & \text { if } & G_{\text {postprandial }}<G_{T}
\end{array} .\right.
$$

Regarding Equation 3, the only unknown variable is $G_{\text {postprandial }}$. However, it could be estimated using a moving average filter and historic postprandial blood glucose data, as:

$$
f(g, N, n)=\frac{1}{k} \sum_{i=0}^{k-1} g(n-i)
$$

where $g(\cdot)$ represents the signal to be filtered, $N$ is the number of observations used in the moving average, $n$ is the most recent observation of the sample to be processed, and $k=n$ if $n<N$ or $k=N$ in other cases. Therefore, the estimated postprandial glycemia for the meal $m$ at day $d$ is represented by $\hat{G}_{\text {postprandial }}^{m}(d)$, and its value is computed as:

$$
\hat{G}_{\text {postprandial }}^{m}(d)=f\left(G_{\text {postprandial }}^{m}, N, d-1\right),
$$

where $G_{\text {postprandial }}^{m}$ represents the real value of the postprandial glycemia for meal $m$. Thus, the estimated bolus for 
meal $m$ at day $d$ is given by:

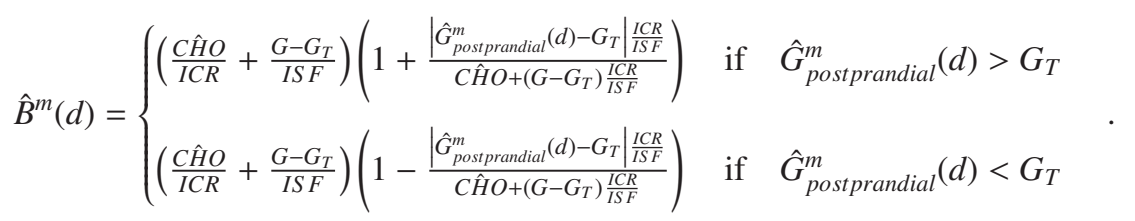

By using Equation 4, it is possible to dynamically adjust the mealtime bolus in order to compensate inaccurate carbohydrate counting and improve the glycemic control of the patients.

\section{DISCUSSION}

To exemplify how to use the proposed bolus calculator let us consider a patient using a total daily insulin dose of $50 \mathrm{U}$ (i.e., $T D D=50 \mathrm{U}$ ), having the following preprandial glycemic target, $G_{T}=110 \mathrm{mg} / \mathrm{dL}$. Assume, without loss of generality, that the $I S F$ and $I C R$ values for this patient are calculated applying the recommendations of the American Association of Clinical Endocrinologists/American College of Endocrinology (AACE/ACE) [8], using the following rules: $I S F=1700 / T D D$ and $I C R=450 / T D D$. Therefore, under these premises, if the patient is planning to eat a meal containing $60 \mathrm{~g}$ of carbohydrates and has a premeal glycemia of $150 \mathrm{mg} / \mathrm{dL}$, it will need to administrate a bolus of about $7.8 \mathrm{U}$ to compensate the meal and correct the high value of the premeal glycemia (n.b., as referred previously, the time between meals is considered to be larger than the duration of the insulin action and, therefore, $I O B=0$ ). However, since patients estimate the carbohydrate content of meals with some error, the postprandial glycemia will present a divergence from the target. Figure 1 presents the correction factor (n.b., according to Equation 3) and the estimated bolus (n.b., using the Equation 4) as a function of the estimated postprandial blood glucose error.

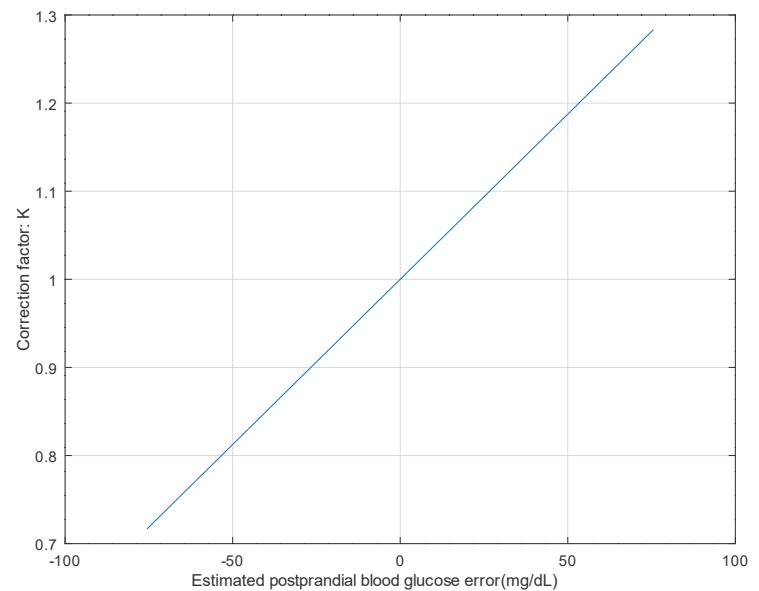

a)

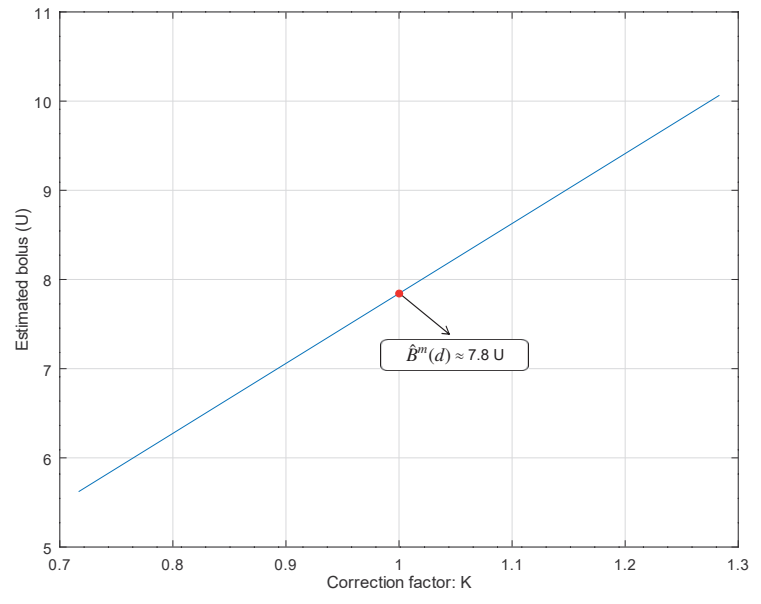

b)

FIGURE 1. a) The correction factor, $K$, as a function of the estimated postprandial blood glucose error, $\left.\hat{G}_{\text {postprandial }}^{m}(d)-G_{T} ; \mathrm{b}\right)$ The estimated bolus, $\hat{B}^{m}(d)$, as a function of the correction factor, $K$.

As can be seen in Figure 1 a), if the postprandial glycemia is on the target, the correction factor is equal to one, on the other hand, if the postprandial glycemia is below or above the target, the correction factor will become smaller or greater than the unity, respectively. On its turn, Figure $1 \mathrm{~b}$ ) shows the estimated bolus, $\hat{B}^{m}(d)$, as a linear function of the correction factor.

\section{CONCLUSION}

Carbohydrate counting is a challenging and error-prone task. In fact, inaccurate carbohydrate counting is common among patients on intensive insulin regimens. As a consequence, these patients frequently experience hypoglycemic 
or hyperglycemic episodes. This work presents an adaptive mealtime bolus calculator designed to compensate for the adverse effects of inaccurate carbohydrate counting. To that end, the proposed bolus calculator uses patient's glycemic data to estimate the value of the postprandial blood glucose and, based on that value, adjust to bolus to be administrated. As future work, this method will be assessed in-silico, using the FDA-approved University of Virginia (UVA)/Padova Type 1 Diabetes Simulator.

\section{ACKNOWLEDGMENTS}

Carlos Abreu and Paula Felgueiras have received a research grant from Ascensia Diabetes Care Portugal under the project: Health Equity for Diabetics: An Intervention for Adults With Type 2 Diabetes in the North Region of Portugal.

This work was supported by Portuguese funds through the CIDMA - Center for Research and Development in Mathematics and Applications, and the Portuguese Foundation for Science and Technology, within project UID/MAT/04106/2013.

\section{REFERENCES}

[1] Walsh J., Roberts R., Bailey T. S., and Heinemann L. Bolus advisors: Sources of error, targets for improvement. Journal of Diabetes Science and Technology, 12(1):190-198, 2018.

[2] Yamada E., Okada S., and Nakajima Y., et al. Effect of carbohydrate counting using bolus calculators on glycemic control in type 1 diabetes patients during continuous subcutaneous insulin infusion. Journal of Diabetes Investigation, 8(4):496-500, 2016.

[3] Deeb A., Al Hajeri A., Alhmoudi I., and Nagelkerke N. Accurate carbohydrate counting is an important determinant of postprandial glycemia in children and adolescents with type 1 diabetes on insulin pump therapy. Journal of Diabetes Science and Technology, 11(4):753-758, 2017.

[4] Smart C. E., Ross K., Edge J. A., Collins C. E., Colyvas K., and King B. R. Children and adolescents on intensive insulin therapy maintain postprandial glycaemic control without precise carbohydrate counting. Diabetic Medicine, 26(3):279-285, 2009.

[5] Smart C. E., King B. R., McElduff P., and Collins C. E. In children using intensive insulin therapy, a 20 -g variation in carbohydrate amount significantly impacts on postprandial glycaemia. Diabetic Medicine, 29(7):21-24, 2012.

[6] Smart C. E., Ross K., Edge J. A., King B. R., McElduff P., and Collins C. E. Can children with type 1 diabetes and their caregivers estimate the carbohydrate content of meals and snacks? Diabetic Medicine, 27(3):348-353, 2010.

[7] Bishop F. K., Maahs D. M., Spiegel G., Owen D., Klingensmith G. J., Bortsov A., Thomas J., and E. J. Mayer-Davis. The carbohydrate counting in adolescents with type 1 diabetes (ccat) study. Diabetes Spectrum, 22(1):56-62, 2009.

[8] Grunberger G., Abelseth J., and Bailey T., et al. Consensus statement by the american association of clinical endocrinologists/american college of endocrinology insulin pump management task force. Endocrine Practice, 20(5):463-489, 2014. 